\title{
El trabajo social en el ámbito laboral desde el abordaje del equipo interdisciplinario
}

\section{Social work in the workplace from the boarding of the interdisciplinary team}

\author{
Benito Danilo Guamán Guamán ${ }^{1 *}$ \\ ${ }^{1}$ Universidad Laica Eloy Alfaro de Manabí, Ecuador \\ *daniloguaman1993@gmail.com \\ DOI: https://doi.org/10.26871/killkanasocial.v4i3.529
}

\begin{abstract}
Resumen
El Trabajo Social en el ámbito laboral cumple un rol sustancial dentro del mundo empresarial, dado que su intervención profesional está enfocada a contribuir al alcance de los objetivos en beneficio de los colaboradores y la empresa, mediante la aplicación de métodos y técnicas propias de la profesión. El objetivo del presente artículo fue identificar el accionar del Trabajo Social desde el abordaje del equipo interdisciplinario para determinar su importancia en el Área de Gestión de Talento Humano de en una empresa atunera del Cantón Montecristi periodo 2019-2020. El tipo de metodología utilizada corresponde a una investigación cuantitativa donde se aplicó encuestas de estructura cerrada y de opción múltiple a todo el personal del Departamento de Talento Humano. Entre los principales resultados obtenidos se contempla el alto índice de la comunicación organizacional interna, comunicación asertiva, el trabajo en equipo, el ejercicio pleno de las trabajadoras sociales en las actividades establecidas, buena estructura organizacional y ejecuciones de reuniones periódicas acorde a las necesidades de cada espacio, lo cual genera que todo el personal conozca cuáles son sus responsabilidades y de los demás. Las principales funciones desempeñadas están direccionadas a acciones preventivas, desarrollo, ejecución y evaluación de proyectos de Bienestar Social, asesoría en trámites del Instituto Ecuatoriano de Seguridad Social (IESS), gestión, orientación y participación en la Responsabilidad Social Empresarial. Por otro lado, todos los profesionales del equipo interdisciplinar reconocen que el abordaje que realiza el Trabajo Social en el Departamento de Gestión de Talento Humano es fundamental para el desarrollo de la empresa atunera y para el equipo interdisciplinario.
\end{abstract}

Palabras clave: trabajo social, ámbito laboral, abordaje, equipo interdisciplinario, talento humano, empresa.

\begin{abstract}
Social Work in the field of work plays a substantial role within the business world, since its professional intervention is focused on contributing to the scope of the objectives for the benefit of employees and the company, through the application of methods and techniques typical of the profession. The objective of this article was to identify the action of Social Work from the approach of the interdisciplinary team to determine its importance in the Area of Human Talent Management of a tuna company in the Canton Montecristi period 2019-2020. The type of methodology used corresponds to quantitative research where closed-structure and multi-choice surveys were applied to all staff in the Department of Human Talent. Among the main results obtained is the high index of internal organizational communication, assertive communication, teamwork, the full exercise of social workers in established activities, good organizational structure and regular meeting executions according to the needs of each space, which means that all staff know what their responsibilities and others are. The main functions performed are directed at preventive actions, development, execution and evaluation of Social Welfare projects, advice on formalities of the Ecuadorian Institute of Social Security (IESS), management, guidance and participation in Corporate Social Responsibility. On the other hand, all professionals of the interdisciplinary team recognize that the approach carried out by the Social Work in the Department of Human Talent Management is fundamental to the development of the tuna company and to the interdisciplinary team.
\end{abstract}

Keywords: Social work, work environment, approach, interdisciplinary team, human talent, company.

\section{Introducción}

El presente artículo tiene como tema central El Trabajo Social en el ámbito laboral desde el abordaje del equipo interdisciplinario. Donde el Trabajo Social empre- sarial realiza su intervención desde el Departamento de Gestión de Talento Humano y también forma parte del equipo interdisciplinario, el cual se encuentra conformado por diversos profesionales de distintas disciplinas. El accio- 
nar del trabajador social con el grupo interdisciplinar está enfocado a realizar su intervención de manera íntegra ante las diversas problemáticas que pueden surgir dentro y fuera de la empresa.

El Departamento de Gestión de Talento Humano está conformado por diferentes áreas que acorde a sus perfiles, por ello surge el interés de determinar la importancia que representa el Trabajo Social desde un enfoque interdisciplinario, de igual forma, identificar si las funciones que ejerce el trabajador social pueden ser realizada por otro experto de distinta especialidad.

El tipo de investigación utilizado corresponde al cuantitativo y la técnica empleada es la encuesta, el cual fue realizado a todo el personal del Departamento de Gestión de Talento Humano de una empresa atunera. Dado que permite la obtención de información de manera numérica y mediante gráficos estadísticos pueden ser descritos y analizados, con la finalidad de llegar a comprobar la hipótesis y a una conclusión.

El objetivo principal está direccionado a identificar el accionar del Trabajo Social desde el abordaje del equipo interdisciplinario para determinar su importancia en el Área de Gestión de Talento Humano de una empresa atunera del Cantón Montecristi periodo 2019-2020, el cual se logró categorizando el grado de comunicación organizacional interna que existe en el equipo interdisciplinario, determinando las funciones que desempeña el Trabajo Social en Talento Humano y finalmente analizando si su accionar como parte del equipo interdisciplinar puede ser realizados por un profesional de otra área.

Finalmente se puede enunciar que la labor del Trabajo Social en el área laboral está vinculado a varias actividades, las cuales están estrechamente relacionadas con la asesoría, gestión, intervención, creación de proyectos, programas y la participación en múltiples funciones que ejerce como parte del equipo interdisciplinario. Además, es importante reconocer la importancia que representa el profesional dentro del Departamento de Gestión de Talento Humano.

\section{Marco Teórico}

\subsection{Definición de Trabajo Social}

Tello (2015) conceptualiza al Trabajo Social como una disciplina que pertenece al campo de las ciencias sociales, donde su principal función o propósito es la intervención social con sujetos específicos, ya sean estos individuales o colectivos que presenten algún tipo de conflicto o problemática social como consecuencia de algún suceso determinado. (p.2)

El autor expone al Trabajo Social como una disciplina que corresponde a las ciencias sociales y abordaje está enfocado a problemáticas sociales que generan vulnerabilidad de individuos o colectivos mediante la aplicación de procesos metodológicos propios de la profesión.

\subsection{Trabajo Social e Intervención}

Falla (2016) expone que la intervención en Trabajo Social depende de dos contextos sociales, la primera es institucional, es decir donde se focalizan los proyectos, planes, políticas de carácter social y la segunda, la intervención profesional como aspecto de poder proporcionar soluciones a los problemas de ámbito individual, familiar, grupal, comunitario. Los dos aspectos son necesarios para poder minimizar los índices de riesgo social. Los trabajadores sociales que ejercen su accionar en los sectores social están destinados a diseñar, ejecutar, gestionar y evaluar proyectos de carácter social que están ligados a las políticas públicas de cualquier gobierno.

Para Saavedra (2015) la intervención social es un conjunto de acciones y actividades desarrolladas de forma holista y organizada, para proceder en una realidad social específica, con la finalidad de producir cambios sustanciales en una determinada problemática social. De igual forma, realza la importancia de la ética profesional al momento de realizar la intervención dirigida a prevenir, cambiar o corregir las problemáticas sociales, mediante los procesos de inclusión social. Uno de los factores principales antes de realizar la intervención, es el diagnóstico social, el en cual el trabajador social debe aplicar técnicas e instrumentos necesarios para la recaudación de información, que permite identificar la raíz de la problemática, con el objetivo de que la intervención pueda ser ejecutada de manera eficaz.

Mientras que para Ibáñez, Olea, Pacheco, Sepúlveda, \& Verdi (2016) la intervención social en Trabajo Social es una herramienta valiosa, dado a que es utilizada para gestionar y promover el mejoramiento de la calidad de vida de la sociedad. Existen dos tipos de intervención a lo largo de la historia, una basada en la intervención objetiva y la otra en una intervención compleja, la primera es entendida como un mecanismo de integración y diálogo que sustentaban la problemática a intervenir, pero desde la dirección de una institución, mientras que la segunda está enfocada en la época actual, debido a que busca identificar a la sociedad antes de realizar una intervención social, esta última tiene como objetivo una integración, reparación y recuperación de la integridad que fueron sustraídas como consecuencia de la desigualdad social. (págs.10-12)

Por lo tanto, la intervención social en Trabajo Social es una operación programada y estructurada metodológicamente direccionada sobre un determinado sector, comunidad, grupo, individuo o familia con el propósito de conseguir cambios sustanciales en sus condiciones de vida. La intervención social conlleva diversas actividades, métodos, técnicas, instrumentos, funciones, tareas que el trabajador social deberá ejecutar para conseguir el objetivo determinado. El fundamento de la intervención social radica principalmente como fase inicial el diagnóstico social que deberá ser realizado de manera minuciosa con la finalidad de identificar la razón inicial de la problemática. 


\subsection{Intervención Profesional en el Campo Laboral}

Para Contreras \& Pilar (2012), la intervención del trabajador social en este campo está relacionado con la gerencia social derivado al desarrollo del talento humano de los colaboradores y del sistema de la seguridad social de todos los que laboran en la empresa, existen muchas áreas dentro del campo laboral donde el profesional puede desempeñar su rol, pero en cualquiera que fuese siempre se basará en el marco de las políticas públicas, los derechos humanos, leyes o reglamento que permitan su accionar. La intervención profesional va dirigida a los problemas internos o externos de los colaboradores. Así mismo, desarrolla diferentes actividades como: programas de capacitación e inducción del nuevo colaborador, visitas domiciliarias, prevención de riesgos laborales, intervención, seguimiento de casos, registro de accidentes de trabajo en las instituciones pertinentes y otras funciones relacionadas con su perfil, así como también ocupaciones asignadas por la organización.

\subsection{Empresa}

Lemon (2016) conceptualiza a la empresa como una estructura organizacional constituida por individuos y recursos que tiene como finalidad lograr ingresos financieros mediante el progreso de una actividad específica. La entidad puede contar con una sola persona en la dirigencia y direccionamiento para lograr alcanzar metas y objetivos previamente estructurados en su planificación empresarial.

\section{Evolución Histórica del Trabajo Social en las Em- presas}

Castillo (2010) expone que el Trabajo Social profesional nación en Inglaterra como consecuencia de la industrialización y sus efectos en nocivos en la calidad de vida de los trabajadores. Aspectos como, trabajo infantil, explotación laboral, carencia de salubridad y falta de seguridad laboral, generaron problemáticas de precariedad social en la población explotada, los cuales ocasionaron que surja la necesidad de un profesional que ofrezca soluciones para mejorarlas condiciones de vida.

Entonces, el paradigma del Trabajo Social empresarial surge a base de todos los problemas socioeconómicos de los trabajadores y sus familias, en primera instancia la profesión cumplía funciones asistencialistas, pero conforme pasó el tiempo fue tomando nuevas responsabilidades orientadas al espacio preventivos.

Para el autor existen tres etapas en la historia del Trabajo Social en la empresa que son:

- Primera Etapa: la Ley de la Beneficencia en 1849 en España, en este periodo surgió la necesidad que las empresas protegieran el capital económico y corregir los errores del capitalismo, sobre todo prevenir una posible revolución laboral en las empresas. El Trabajo Social empresarial nace como estrategia para controlar a los trabajadores y a sus familias, la función del profesional estaba enmarcada en exponer los beneficios que la empresa ofertaba al trabajador, tales como: servicio médico y educativo, caja de retiro, esto el sector empresarial lo ejecutaba por medio de prestaciones sociales a cambio de fidelidad, lealtad y esfuerzo.

- Segunda Etapa: El Trabajo Social en etapa inicia en los primeros años del franquismo y culmina al final de la misma época. Durante este periodo el estado comienza a regular a las empresas para que ofrezcan a sus trabajadores los siguientes servicios: comedores para los trabajadores, el economatos y servicio de salud.

- Tercera Etapa: Comienza con los inicios de la democracia y el establecimiento de redes públicas, que tuvieron una repercusión en las empresas, donde éstas debían ofrecer servicios de bienestar ocupacional al trabajador y velar por el beneficio propio de la empresa, el objetivo fundamental radica la prevención de accidentes laborales y asistir en caso de que exista alguna lesión o afectación de la salud del trabajador en el puesto de trabajo, el propósito era generar un ambiente seguro y saludable a todos los colaboradores de la empresa. En esta forma, el trabajador social se complementará con el equipo interdisciplinar para abordar de forma total las necesidades de la empresa y trabajadores. (Castillo, 2010, pp.75-81)

La evolución del Trabajo Social en empresa comienza en dejar atrás la asistencia social que sus inicios no fueron tan claros, para dar paso a la profesión de carácter objetiva debido a que las empresas comenzaron a desarrollar programas y proyectos de responsabilidad social. Por ello, la profesión se posesionó de carácter estratégico dentro del Departamento de Gestión de Talento de una organización para desempeñar funciones en bienestar de los trabajadores, la familia y de la empresa. Además, forma parte del equipo interdisciplinar con la finalidad de abordar de manera íntegra las necesidades de los colaboradores.

\section{Trabajo Social y Empresa}

Cuerda \& Olarte (2015) hace referencia que el Trabajo Social empresarial surgió desde una perspectiva asistencialista, donde su función era detallar los beneficios que oferta la empresa hacia sus colaboradores, pero conforme pasaron los años, la necesidad de las industrias fueron aumentando, aún más cuando la responsabilidad social surgió como aspecto central de la organización y sus colaboradores, así mismo aporta con su accionar en campos de la salud ocupacional, seguridad social, gestión de cambio, conciliación personal, familiar, laboral, aspectos diversidad, entre aportaciones, donde su función se centra o responde a las necesidades de los colaboradores y de la organización. (p.58)

Para Raya \& Caparrós (2013) el nuevo escenario de intervención profesional está relacionado al ámbito empresarial o laboral, misma que surge en España como consecuencia de la industrialización y la define como una acción organizada, que busca ayudar a la adaptación mutua de los colaboradores y su empresa. 
Para el autor la función del trabajador social está articulado en tres dimensiones fundamentales que son:

- Brindar apoyo psicosocial a los colaboradores que presenten algún tipo de dificultad.

- Contribuir en la creación y ejecución de las acciones sociales en beneficio de los colaboradores y la empresa.

- Asistir a un mejor entendimiento de los conflictos humanos y sociales surgidos como consecuencia del trabajo.

El Trabajo Social en la empresa cumple diversas funciones en la participación y trabajo en equipo con los demás departamentos que conforman una empresa, mismas que son:

1) La colaboración del trabajador social con el Comité de Empresa,

2) La participación en la mejora del ambiente empresarial y extra empresarial que influyen en las causas de enfermedad.

3) Fomentar la adquisición de una conciencia social entre el personal, ayudando a superar todas las causas de absentismo. (Raya y Caparrós, 2013, p.5)

Las funciones que ejerce el trabajador social tienen relación directa con las propuestas realizadas por los autores. Aunque en la actualidad han surgido nuevas actividades en las cuales puede realizar su función, una de ellas, es la prevención de riesgos de trabajo que a sus inicios que no era parte de la competencia del Trabajo Social, pero se ha ido convirtiendo en una de las ocupaciones por el cual el profesional es contratado.

Mientras que para Urra (2013) el objetivo del Trabajo Social en la empresa es, diseñar procesos que generen desarrollo empresarial, sobre todo que tengan un impacto en la productividad, mediante la compresión de los factores socioculturales de la empresa y de sus colaboradores, de igual forma, su función también implica el desarrollo, ejecución y evaluación de programas de ámbito social.

Finalmente, las definiciones es propuestas por los autores conllevan una relación coherente en la el accionar del Trabajo Social en la empresa, por lo tanto, se puede conceptualizar como aquella actividad organizada que tiene como finalidad buscar la adaptación recíproca de los colaboradores en la empresa, mediante la aplicación de métodos, técnicas e instrumentos que les permitan hacer frente a las problemáticas sociales e individuales que impidan su ajuste. Además, busca generar un bienestar económico y social de los trabajadores, a través de la potencialización de los recursos humanos.

\subsection{Departamento de Talento Humano y Trabajo Social}

Para Chaparro \& Urra (2013) el Trabajo Social se ha desarrollado de una manera estratégica dentro de las organizaciones, donde el capital humano es el valor más importante en una empresa, es ahí donde el profesional cumple múltiples funciones para brindar una buena calidad de vida personal, laboral y familiar. Las ocupaciones que ejerce están ligadas al cumplimiento de metas y objetivos, mediante el desarrollo de estrategias en concordancia con los lineamientos de la organización, mismas que son: Participación en el desarrollo de políticas en el Departamento de Gestión de Talento Humano, dirección del personal de la empresa, desarrollo del colaborador, mediante la motivación para conseguir los objetivos de la empresa, fomentar la participación de los colaboradores en la organización y mejora del bienestar laboral de los colaboradores.

Las múltiples actividades que ejerce el área de Trabajo Social en el Departamento de Gestión de Talento Humano, está encaminado a contribuir al logro de las metas en base al perfeccionamiento de las potencialidades de los colaboradores, mediante la implantación de capacitaciones, motivaciones, incentivos y demás estrategias que permitan alinear los sueños de los trabajadores con la empresa.

Mientras que Sáenz (2015) alude que el Trabajo Social se ha incursionado estratégicamente en el área de Talento Humano, donde se ha involucrado en la planificación y desarrollo de entrevistas de preselección de nuevos colaboradores, toma de decisiones a nivel macro institucional sobre el clima laboral y calidad de vida que oferta la empresa. De igual manera, se realza la participación y el trabajo del profesional en equipos interdisciplinarios, así como también en la labor que centra en la ejecución de programas de reclutamiento, selección, inducción, entrenamiento y capacitación al nuevo trabajador y cuidado microsocial, que en algunas empresas se ha convertido en un tema estratégico para el desarrollo empresarial.

Conforme los enfoques de los dos autores, se puede concluir que ambos tienen una visión diferente al accionar del Trabajo Social en el Departamento de Talento Humano, pero si algo entrelaza las dos definiciones es la búsqueda del bienestar del trabajador y de la empresa. Del mismo modo, la profesión se ha adjudicado estructuradamente en nuevos campos de intervención social, en el cual su rol ha trascendido de manera crucial, dado que su objetivo se ha centrado no solamente en el reclutamiento, selección, contratación, inducción y capacitación del nuevo personal, sino, en la intervención en el clima laboral de la empresa, cuya función permite diagnosticar e implementar estrategias que mejoren las relaciones entre los colaboradores.

\section{2 Áreas de Intervención del Trabajo Social en el Ámbito Laboral}

Raya \& Caparrós (2013), expresa que las áreas de intervención del Trabajo social están enfocadas en cuatro aspectos sustanciales que son:

- En la Salud Laboral: La intervención en esta área está enfocada en prevenir y orientar a los colaboradores en circunstancias de adicción, otra de las funciones es el direccionamiento al personal para el desarrollo de habilidades y destrezas, de igual modo, detectar las circunstancias que generen estrés en los trabajadores, con la finalidad de buscar mecanismos, técnicas y estrategias encaminadas a dar soluciones a dichas problemáticas. Esto puede ser ejecutado de manera conjunta con otros 
departamentos que integran el departamento de Talento Humano. (Raya y Caparrós, 2013)

- En las Relaciones Profesionales: En este campo el ejercicio profesional está destinado al estudio del clima laboral, en la prevención, mediación y resolución de conflictos, sobre todo, intervenir en casos de absentismo laboral. (Raya y Caparrós, 2013)

- En el Contextos Personal y Familiar de los Colaboradores: La actuación del Trabajo Social tiene que ver con el cuidado social de la familia del colaborador, a través de la información y disposición sobre los recursos internos y externos de la empresa, esto está afín a la ayuda social y otros mecanismos de cooperación a los trabajadores. (Raya y Caparrós, 2013)

- En la Proyección Social y Responsabilidad Social Empresarial: Este aspecto es uno de los más importante en el cual el trabajador social se desenvuelve en el ámbito laboral, dado que su intervención o función va direccionada a diseñar programas o proyectos de voluntariado, de la misma manera, diseña, tramita y evalúa los proyectos de responsabilidad social, al igual que los planes de conciliación familiar y laboral de los colaboradores. (Raya y Caparrós, 2013)

Del tal modo que la intervención del trabajador social en el contexto empresarial puede variar dependiendo a las funciones o departamentos que las organizaciones designen al profesional, están pueden ser, departamento psicosocial, departamento de prevención de riesgos laborales o directamente con el departamento de Talento Humano. Cualquiera que fuera el área de asignación del trabajador social, siempre estará en contacto continuo con lo colaboradores, sobre todo trabaja juntamente con los diversos equipos interdisciplinarios de la empresa.

\subsection{Departamento de Talento Humano}

Para las autoras Vallejo \& Portalanza (2017) el Talento Humano a lo largo de la historia ha pasado por grandes cambios, pero en la década de los 90 recién adquiere un realce sustancial en cuanto a la gestión organizacional, donde además adquiere nuevas competencias y responsabilidades en los proceso que ejecutan como departamento. Por ello, la Gestión de Talento Humano en la actualidad es sustancial en el ámbito empresarial, dado que depende de las estrategias y operaciones que se planifican para lograr un buen desenvolvimiento laboral, teniendo en cuenta diversas variables como son: la cultura, aptitudes, actitudes, habilidades y destrezas de cada uno de los miembros que conforma el área.

Mientras que Blacutt (2013) expresa que el Departamento de Talento Humano como tal se encarga de planificar, diseñar, planificar, coordinar, ejecutar y la prestación de servicios administrativos de los colaboradores como:

- Preparación y alineación del trabajador a la empresa.

- Bienestar social.

- Seguridad y salud ocupacional.
Todo aquello basados en las directrices, normativas y políticas legales de la empresa en concordancia con las leyes que rigen en el Ecuador.

\subsection{Funciones de Talento Humano}

Las diversas funciones que realiza el departamento de Talento Humano en el ámbito empresarial son:

\subsubsection{Diseño de Cargos}

Esta función es trascendental en el cual la Gestión de Talento Humano debe colocar todo el interés para realizarlo, dado que, en ello se engloba las responsabilidades y actividades que los colaboradores deben realizar, evitando malestares y cansancio innecesario o redundantes. El diseño de los cargos debe ser desarrollados a tal grado que se englobe al colaborador como parte crucial dentro de la empresa, sobre todo para generar que el cumple un rol en importante en el alcance de los objetivos institucionales. Con la finalidad de generar motivación y colaboración a las funciones que ejerce dentro de la organización, como consecuencia el rendimiento laboral crecerá sustancialmente. (Vallejo y Portalanza, 2017)

\subsubsection{Reclutamiento y Selección}

Esta función genera un desafío o reto para el departamento de Talento Humano, dado a que se ejecuta antes de la incorporación del nuevo personal a la empresa, es decir, el reclutamiento, en el cual existen diversos mecanismos para captar al personal potencial para el puesto que requiere la organización, una de ellas, es el reclutamiento interno y externo, el primero se fundamenta en búsqueda del personal que cumpla con el perfil de manera interna en la empresa, el segundo se caracteriza por la búsqueda del personal por diversos aspectos, ya sea físicos o por los medios de comunicación como: periódicos, páginas web para empleos, medios radiales, cuñas comerciales, entre otras. En el cual se dispone el cargo y el perfil que debe cumplir el aspirante. Una vez adquirido todas las carpetas o hojas de vida de los postulantes, ya sea de carácter físico o digital se procederá a seleccionar a persona que más se asemeja al perfil del puesto solicitado y procederá a la firma de contrato. (Vallejo y Portalanza, 2017)

\subsubsection{Capacitación}

La capacitación en el entorno de Talento Humano ha ido evolucionando a lo largo del tiempo, logrando así que se definida como una actividad constituida y organizada para la generación de cursos, talleres y capacitaciones, donde se estipula los horarios, metas y objetivos a alcanzar. Con el propósito de dotar al personal, herramientas conocimientos, aptitudes, actitudes y habilidades necesarias para que puedan ejercer de manera eficaz el trabajo asignado en cada una de las áreas. Por ello, la capacitación y el desarrollo profesional es uno de los temas en el cual el departamento de Talento Humano debe poner todo su interés, ya que generará beneficios sustanciales para la organización empresarial. (Vallejo y Portalanza, 2017) 


\subsubsection{Evaluación de Desempeño}

La evaluación del desempeño es uno de los temas cruciales para el departamento de Talento Humano, ya que mediante ello se puede detectar posibles problemáticas para poder abordarlos oportunamente y así poder mejorar la calidad del recurso humano y, por ende, se incrementará la productividad. El proceso de evaluación del desempeño se ejecuta a través de medición del grado de desempeño de los objetivos y del desarrollo de valores de los colaboradores, que pueden estar direccionado a los procedimientos de exploración salarial y estímulos a los trabajadores. Dado que un personal motivado y estimulado implica mayor productividad para la empresa, del mismo modo, permite detectar a aquellos colaboradores con gran potencial y de esta forma desarrollar y diseñar planes de sucesión que beneficiará económicamente a la organización. (Vallejo y Portalanza, 2017)

\subsubsection{Remuneraciones, Compensaciones y Beneficios}

Se miden dependiendo de los indicadores de impacto de los niveles de motivación y compromiso de los colaboradores con la empresa, esto resalta la necesidad de crear lazos firmes entre los trabajadores y la institución, mediante la elaboración de planes y políticas flexibles con el objetivo facilitar la conciliación personal y laboral, existen dos tipos de compensación, la primera es monetaria que puede estar relaciona o no con el salario, la segunda, no monetaria, que representa al clima laboral, bienestar laboral, comunicación interna y trabajo en equipo. (Vallejo y Portalanza, 2017)

\subsubsection{Planes de Sucesión}

La elaboración de planes de sucesión se ha convertido en un eje clave del departamento de Talento Humano, destinado a detener y atraer a los colaboradores más eficaces. Las empresas deben optar por estos cambios para mejorar las condiciones laborales y así hacerlo más flexible para comprometer al colaborador, la sucesión en términos actuales se ha convertido en uno de los retos más importantes para el siglo XXI, dado que persigue la permanencia, desarrollo y la prolongación de la empresa a lo largo del tiempo. (Vallejo y Portalanza, 2017)

\subsection{Equipo Interdisciplinario}

UtopiaCF (2017) describe que el mundo empresarial internamente existe dos equipos que buscan lograr las tareas y actividades de la organización, el primero el equipo multidisciplinar y el segundo, el equipo interdisciplinar, mimas que está conformada por profesionales de distintas disciplinas que aportan con sus conocimientos, habilidades y destrezas para el buen funcionamiento del equipo. La objetividad del equipo interdisciplinar otorga a los miembros aspectos como la creatividad, dinamismo, trabajo en equipo, entre otras cualidades que representa la esencia alteradora. Aunque sus funciones son semejantes al del equipo multidisciplinar, este le da dos valores agregados, la primera es la conexión de ideas mediante la creatividad y el valor agregado viene siendo el trabajo de condición conjunta, donde todos colaboren de manera eficaz y la segunda, es la importancia y el realce de la comunicación entre los miembros.

El mismo autor, describe las que existen cuatro ventajas sustanciales que son:

\subsubsection{Ventajas del Equipo Interdisciplinar}

- La metodología que se despliegan en un equipo interdisciplinario es tremendamente creativa que aumentan las posibilidades del éxito.

- El trabajo en equipo interdisciplinario genera espacios para el diálogo e intercambio de información.

- La multiplicidad y la primicia son pilares fundamentales del equipo.

- Intercambio de aprendizajes, donde cada profesional comparte sus conocimientos en cuanto a su especialización y de la misma manera adhieren habilidades de otros profesionales.

\subsection{El Trabajo Social como Parte del Equipo Interdisci- plinar}

Para Solana (Solana, 2016) el tema de interdisciplinaridad en Trabajo Social logró notoriedad públicamente en la década de 1970, donde se define que la intervención social puede darse en distintas áreas, a base de aspectos metodológicos y epistemológicos. Donde el abordaje interdisciplinar va encaminado a la solución de los problemas con carácter de complejidad, los cuales se intervienen de forma compartida con las demás disciplinas que integran en una organización. El Trabajo Social al igual que los profesionales de las demás disciplinas deberán aplicar procesos metodológicos para abordar el problema de condición agregada, donde cada uno aporte con sus conocimientos del campo específico.

Mientras que Robles (Robles, 2017) expone que el Trabajo Social en el contexto interdisciplinar está encargado de abordar los problemas o necesidades de los colaboradores de manera conjunta con los profesionales que integran Talento Humano, con la finalidad de abordarlo de forma eficaz. Para ello, el apoyo del equipo interdisciplinar es fundamental para lograr, no solamente intervenir el problema, sino también alcanzar los objetivos propuestos por la empresa.

Es evidente que el equipo interdisciplinar en cual el Trabajo Social forma parte requiere de un apoyo constante de cada una de las distintas disciplinas, dado que su abordaje es de carácter integral, donde no solo se limita al colaborador o a lograr los objetivos de la empresa, sino, da un valor agregado en proceso de aplicación, el cual consiste en la utilización y aplicación de procesos metodológico al momento de intervenir una problemática compleja. 


\subsection{Modelo de Intervención del Trabajo Social en el Campo Laboral}

Existen varios modelos de intervención social que pueden ser aplicados en el campo empresarial, pero por la naturaleza de la investigación se hará referencia dos de ellos que son:

Modelo de Gestión de Casos: La intervención social desde este modelo es integra y eficiente, sobre todo puede ser a corto y largo plazo con la finalidad asegurar que los usuarios que presente múltiples problemáticas reciban un servicio de manera adecuada y acorde a sus necesidades. El abordaje del Trabajo Social es de manera directa (individuo-familia), y de manera indirecta (organizaciónempresa) a través de programas direccionados a organizaciones y políticas pública que representa a la dimensión microsocial, en el cual, su accionar no solo está ligado al usuario, sino también al contexto donde desenvuelve con la finalidad de mejorar las condiciones su condición de vida. Finalmente, el modelo de gestión de caso no requiere de manera explícita la aplicación de técnicas y procesos de intervención específicas, pero dependerá su utilización dependiendo los niveles directo e indirecto. Así mismo, el trabajador social deberá aplicar habilidades, destrezas, capacidades y aptitudes profesionales durante el proceso de intervención (Viscarret, 2017, p. 246).

Modelo Sociolaboral: La intervención social desde este modelo tiene con base central determinar las necesidades de los colaboradores, su familia y comunidad, con el propósito de mejorar sus condiciones de vida en base a dos parámetros de intervención, la primera la orientación sociolaboral que está vinculado en el abordaje de casos individuales o colectivos y el segundo la inserción sociolaboral con el soporte de la economía social y cooperativa para superar la vulnerabilidad y conseguir una unificación autónoma y práctica conocida como el tercer sector (Fernández, 2017).

\subsection{Código de Trabajo}

El Ministerio del Trabajo (2018), en su Registro Oficial Suplemento 167 de 16-dic-2005 y última modificación 26-sep-2018 que se encuentra vigente en la actualidad establece lo siguiente: Art. 42.- Obligaciones del empleador.Son obligaciones del empleador: [...] 24. La empresa que cuente con cien o más trabajadores está obligada a contratar los servicios de un trabajador social titulado. Las que tuvieren trescientos o más, contratarán otro trabajador social por cada trescientos de excedente. Las atribuciones y deberes de tales trabajadores sociales serán los inherentes a su función y a los que se determinen en el título pertinente

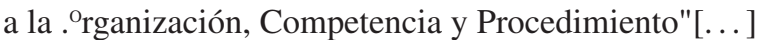

\section{Metodología}

\subsection{Tipo de Investigación}

El tipo de investigación del presente proyecto es de tipo cuantitativo porque proporciona la aplicación de encuestas que a su vez arrojarán gráficos estadísticos que ofrecen datos reales de la situación a investigar, de igual manera, otorga un valor numérico que permite recopilar, describir y analizar la información. De investigación bibliográfica porqué posibilitó la búsqueda y recopilación de información por medio de la indagación en libros físico y digitales, artículos científicos, artículos de revista, sitios web, entre otros y finalmente la investigación de campo porque condesciende el acercamiento con los profesionales que integran el equipo interdisciplinar del Departamento de Talento Humano de la empresa atunera.

\subsection{Diseño de Investigación}

Los diseños de investigación que fueron utilizados en el presente proyecto de investigación son: Exploratorio porque contempla la primera fase que se llevó a cabo, y facilitó conocer e identificar el contexto en que se desarrolló el estudio y descriptivo para describir la importancia del Trabajo Social en el ámbito laboral, dentro equipo interdisciplinario del departamento de Talento Humano, por medio del detalle de los datos que se obtuvieron en la aplicación de la encuesta, con el propósito de analizarlo e interpretarlo y llegar a una posible conclusión.

\subsection{Métodos de Investigación}

En investigación desarrollada se emplea los métodos: Analítico para recopilar, examinar y analizar los datos obtenidos de la encuesta mediante cuadros estadísticos, para evidenciar la idea central del tema de estudio y explicativo fundamento que permitió describir la importancia del Trabajo Social en el ámbito laboral, dentro del equipo interdisciplinario de la empresa atunera.

\subsection{Población y Muestra}

La presente investigación tiene como referencia a 26 colaboradores del Departamento de Talento Humano de una empresa atunera del cantón Montecristi que representan a: Jefe de Talento Humano, Seguridad industrial, Trabajo Socia, Psicología, Nómina, Asistentes, Dispensario Médico, Reglamento Interno y Comunicación

\subsection{Técnicas e Instrumentos de Investigación}

La técnica empleada fue la encuesta porque proporcionó la exploración sistémica de la información, con objetivo de recolectar datos de manera individual y el cuestionario porque permitió la formulación de preguntas cerradas dicotómicas y preguntas cerradas con múltiples opciones, lo que facilitó la recolección de datos necesarios para establecer la importancia del Trabajo Social en ámbito laboral, dentro del abordaje interdisciplinario de la empresa atunera.

\section{Resultados}

Entre las 11 preguntas de la encuesta establecidas a los diversos profesionales del equipo multidisciplinario de una empresa atunera es la primera que hace relación al nivel de 
comunicación organizacional interna en el equipo interdisciplinar del Departamento de Talento Humano en el cual del $100 \%$ de los encuestados, el $76.9 \%$ expresa que el nivel de comunicación organizacional interna es alto, mientras que el $23.1 \%$ manifiestan que es medio. Donde el personal del equipo interdisciplinario que conforma el Departamento de Talento Humano afirma en su gran mayoría que la comunicación organizacional interna es alta, lo que representaría que en las áreas existe la transferencia y distribución de la información de manera asertiva y colaborativa con todos los profesionales de las distintas disciplinas.

La segunda pregunta está direccionada a determinar la frecuencia que se reúne el personal que conforma el Departamento de Talento Humano, donde el $38.5 \%$ del $100 \%$ del personal que conforma el Departamento de Talento Humano ostentan que se reúnen cada mes, seguido del $23.1 \%$ cada semana, el $19.2 \%$ cada quince días y con el mismo valor de $19.2 \%$ más de un mes. Esto se realiza en base a las distintas necesidades de cada una de las áreas, dado que disponen de planificaciones o cronogramas previamente establecidos que enmarcan las reuniones periódicas que amerite la participación e intervención de los profesionales de las distintas disciplinas que forman el equipo interdisciplinario de la empresa atunera.

La tercera pregunta fue: ¿Es fundamental el trabajo en equipo para el desarrollo del Departamento de Talento Humano? Como respuesta el $100 \%$ del personal que integral el equipo interdisciplinario enuncian que el trabajo en equipo es importante para el desarrollo del Departamento de Talento Humano, dado que contribuye de manera rápida y eficaz al alcance de las metas y objetivos propuestos por la organización, mediante la colaboración, creatividad y compromiso de cada uno de los profesionales que componen las diversas áreas, lo que significaría que el personal está alineado a la misión y visión de la empresa atunera.

La cuarta pregunta plantea es: ¿La comunicación asertiva entre las distintas áreas influye de manera positiva en la empresa? El $100 \%$ de los encuestados que son parte de equipo interdisciplinar del Departamento de Talento Humano enuncian que comunicación asertiva si influye de manera positiva entre las distintas áreas de la empresa. Dado que la comunicación asertiva contribuye de manera positiva a la organización, ya que mediante ella se pueden comunicar de forma clara, honesta, concreta y con respeto, en los cuales estos aspectos influyen de manera directa en el rendimiento y desempeño del personal, sobre todo aporta de forma positiva al alcance de los logros y objetivos proyectados para alcanzar el éxito empresarial y, por ende, el de sus colabores y sus familias.

La quinta pregunta corresponde a que si el equipo interdisciplinario conoce cuáles son las funciones del área de Trabajo Social en el Departamento de Talento Humano, donde el $100 \%$ de los profesionales del equipo interdisciplinario del Departamento de Talento Humano mencionan que, si conocen las funciones del área de Trabajo Socia en beneficio de los colaboradores y de la empresa. Esto concierne una vez más a la comunicación en la estructura o jerarquía organizacional, donde cada uno de ellos conocen las funciones o roles que realizan cada profesional en sus respectivas áreas, de igual modo las reuniones periódicas que mantienen facilitan la fluidez de la información, así mismo el direccionamiento eficiente que realiza la Gerente de Talento Humano.

La sexta pregunta está direccionada a determinar cuáles son las funciones del Trabajo Social en el Departamento de Talento Humano donde los 26 profesionales del equipo interdisciplinar del Departamento de Talento Humano tienen la congruencia de elegir varias opciones como respuesta, en el cual se establece que las funciones del área de Trabajo Social son: con un $11.9 \%$ es la orientación a los colaboradores en trámites del Instituto Ecuatoriano de Seguridad Social, en adelante se usará las siglas IESS, el $10.3 \%$ velar por los derechos de grupos de atención prioritaria (mujeres embarazadas y personas con discapacidad), $10.3 \%$ que participa en la Responsabilidad Social Empresarial, el $10.3 \%$ que participa en la gestión de Talento Humano, $8.8 \%$ atención a la diversidad, el $8.8 \%$ que mantiene un clima laboral accesible entre los colaboradores y la empresa, un $8.8 \%$ elaboración, ejecución y evaluación de proyectos sociales en el Departamento de Bienestar Social, un $8.3 \%$ que participa en la prevención de riesgos psicosociales, el $8.3 \%$ que participa en mediación laboral, un $7.8 \%$ Conciliación personal-laboral, el $5.4 \%$ que desarrolla y ejecuta planes de igualdad de derechos laborales , mientras que el $0.5 \%$ participa en prevención de riesgos de trabajo y finalmente, con el mismo valor, $0.5 \%$ que realiza capacitación a los colaboradores. En el cual la respuesta positiva a esta interrogante está relacionada a los anteriores resultados, dado que la estructura organizacional, la comunicación y el trabajo en equipo influyen de forma positiva a que el personal conozca las actividades que desenvuelve cada área con el propósito de contribuir al alcance de las metas y objetivos de la empresa atunera.

La séptima pregunta es: ¿Las actividades y funciones que realiza el departamento de Trabajo Social son importantes para la empresa? y como resultado el $100 \%$ del personal que conforma el equipo interdisciplinar de Talento Humano, manifiestan que las actividades y funciones que realiza el departamento de Trabajo Social si son importantes para la empresa y aquello es por la eficiente labor que ejercen las trabajadoras sociales para contribuir de carácter positivo al alcance los propósitos planteados, mediante la aplicación de modelos, técnicas y procesos de intervención social que aporten al cumplimiento de los objetivos en beneficio a los colaboradores y a la organización.

La octava pregunta es: ¿Considera que es fundamental la participación del Trabajo Social en el proceso de planificación, organización y prevención? en el cual el $100 \%$ del equipo interdisciplinar de Talento Humano considera que, si es fundamental la participación del Trabajo Social en el proceso de planificación, organización y prevención Esta generalidad puede estar asociada a la eficaz intervención 
que realizan las trabajadoras sociales dentro de su área, ya que su abordaje puede estar direccionado a la prevención de riesgos de trabajo y psicosociales, en la planificación y organización de proyectos sociales, que benefician al desarrollo organizacional de la empresa atunera.

La novena pregunta es establecer si accionar del Trabajo Social como parte del equipo interdisciplinar de la empresa genera impacto positivo donde el $100 \%$ del personal encuestado del Departamento de Talento Humano, expresan que accionar del Trabajo Social como parte del equipo interdisciplinar de la empresa si genera impacto positivo y aquello puede está relacionado con la oportuna intervención profesional que ejecutan las trabajadoras sociales con el grupo, ligado al beneficio de los colaboradores y al desarrollo empresarial.

La décima pregunta se enfoca en identificar ¿Qué tan importante es el accionar del Trabajo Social como parte del equipo interdisciplinario del Departamento de Talento Humano? y con un $92.3 \%$ de los encuestados enumeran que el accionar del Trabajo Social como parte del equipo interdisciplinario del Departamento de Talento Humano es alto, pero el $7.7 \%$ ostentan que es medio. Esta afirmación se relaciona directamente con la pregunta anterior, en cual el abordaje de las trabajadoras sociales como miembro del equipo interdisciplinar genera un impacto positivo y su contribución es importante para el grupo, dado que las funciones que ejercen de manera conjunta esta direccionado a una intervención integra y oportuna de las diversas problemáticas internas existentes, para crear un acontecimiento afirmativo para la empresa atunera.

La onceava pregunta se encamina a determinar si el accionar del Trabajo Social como parte del equipo interdisciplinar, podrá ser realizado por otro profesional del Departamento de Talento Humano y como resultado el $53.8 \%$ manifiestan que el accionar del trabajo Social como parte del equipo interdisciplinar no puede ser realizado por otro profesional, frente a un $46.2 \%$ que enuncian que si puede ser ejercido por otro personal del Departamento de Talento Humano. El valor de esta respuesta coincide con el trabajo colaborativo y en equipo. Aunque del mismo modo, dependería de la designación que realice el/la jefa/e inmediato en cuanto a la colaboración de otro profesional ajeno al Trabajo Social de manera transitoria dependiendo de la necesidad o requerimiento del sector, pero siempre enfocado al beneficio de la organización, más no a cumplir sus funciones en su totalidad.

\section{Conclusiones}

En base a los resultados obtenidos, se afirma que el accionar del Trabajo Social desde el abordaje del equipo interdisciplinario es importante porque genera un impacto positivo, sobre todo que las funciones que ejerce en el área no pueden ser asumida por otro profesional de una distinta disciplina del Departamento de Gestión de Talento Humano de la empresa atunera.
El grado de comunicación organización interna dentro del equipo interdisciplinario es alto y como consecuencia de ello surge la prominente participación del personal para el trabajo en equipo y el intercambio de información de carácter asertivo, pero no solo se encuentra presente en el personal, sino también en la alta gerencia, dado que cada uno de los miembros que integran las distintas áreas conocen las funciones que realiza cada una de ellas para contribuir al logro de las metas y objetivos del Departamento de Talento Humano y de esta manera aportar al desarrollo organizacional de la empresa atunera.

Las funciones de manera generalizada del Trabajo Social son: identificar, diagnosticar, orientar en trámites del IESS e intervenir. Además, desarrolla, ejecuta y evalúa proyectos, planes o programas relacionado con la Responsabilidad Social Empresarial y Bienestar Social. También aspectos: económicos, psicológicos, psicosociales, sociales, capacitaciones, relaciones productivas y relaciones humanas, en beneficio de la empresa y de los colaboradores.

Se determina que el accionar del Trabajo Social desde el abordaje del equipo interdisciplinario es importante, dado que su función está encaminada al beneficio de los colaboradores y al logro de los objetivos del Departamento de Gestión de Talento Humano de la empresa atunera.

Finalmente, el accionar del Trabajo Social dentro del equipo interdisciplinar no puede ser asumido en su totalidad por otro profesional de una distinta disciplina. Sin embargo, el personal pude apoyar la gestión que realizan las trabajadoras sociales en su área mediante el trabajo en equipo, aunque la designación dependerá del jefe inmediato en base a las necesidades y requerimiento detectados en los diversos espacios del Departamento de Talento Humano.

\section{Recomendaciones}

Que, se continúen potencializando la comunicación organizacional interna, comunicación asertiva y el trabajo en equipo, dentro del equipo interdisciplinario del Departamento de Talento Humano, a pesar de que los resultados fueron satisfactorios es importante seguir fortaleciendo el diálogo e intercambio de información de forma eficaz, dado que es un factor trascendental para continuar con el desarrollo de la empresa atunera.

Que, el área de Trabajo Social siga desarrollando su abordaje conforme su rol profesional en el ámbito laboral, ya que su accionar representa un valor crucial para el equipo interdisciplinar del Departamento de Talento Humano, sobre todo aporta al alcance de las metas y objetivos en beneficio de la empresa atunera, sus colaboradores, familia y sociedad.

Que, las funciones que realiza el Trabajo Social dentro del área no sean asumidas de manera total por otro profesional de una distinta disciplina, sino más bien, proseguir impulsando el trabajo en equipo en base a las necesidades y requerimientos que lo amerite o lo establezca la Gerente de Talento Humano en el beneficio de los colaboradores y de la empresa atunera del cantón Montecristi. 
Blacutt, M. (2013). El Desarrollo Local Complementario - Libro Gratis. España: Fundación Universitaria Andalu.

Castillo, A. (2010). EL IMAGINARIO DEL TRABAJO SOCIAL EN LAS TESINAS DE FIN DE ESTUDIOS 1938-1983 (Primera edición ed.). España: Cicegraf, S.L.

Chaparro, M., y Urra. (2013). Trabajo social en la gestión del talento humano: de lo operativo a lo estratégico. Perspectivas, 24, 145-157. doi: 10.29344/07171714 .24 .480

Cuerda, I. D. V., y Olarte, E. T. (2015). Presente y futuro del trabajo social en la empresa privada. Documentos de trabajo social: Revista de trabajo y acción social(56), 52-63.

Falla Ramírez, U. (2016, jun). La intervención como forma de poder en el trabajo social. Tabula Rasa(24), 349368. doi: $10.25058 / 20112742.69$

Ibáñez, R., Olea, T., Pacheco, C., Sepúlveda, M., y Verdi, J. (2016). INTERVENCIÓN Y RE- PRODUCCIÓN EN EL TRABAJO SOCIAL. REGISTRO DE PROPIEDAD INTELECTUAL $N^{\circ} 265476,58$.

Lemon, A. (2016). Empresa - Definición, qué es y concepto.

Raya, E., y Caparrós, N. (2013). Trabajo social en las relaciones laborales y la empresa: vías para el emprendimiento., 52, 19.

Robles, K. (2017). El ejercicio profesional de Trabajo Social dentro de los Equipos Interdisciplinarios del programa PROMECUM en Costa Rica. Universidad de Costa Rica, Sede Occidente, 84, 12.

Saavedra, J. (2015). Cuatro argumentos sobre el concepto de intervención social. , 12.

Sáenz, J. (2015). TRABAJO SOCIAL EN RECURSOS HUMANOS. Universidad de Costa Rica, 8.

Solana, J. (2016). TRABAJO SOCIAL, COMPLEJIDAD E INTERDISCIPLINARIEDAD: UNA SÍNTESIS DE ALGUNOS PLANTEAMIENTOS EN ESPAÑA E HISPANOAMÉRICA. , 2, 14.

Tello, N. (2015). Apuntes de Trabajo Social (Tercera Edición ed.). México: Estudios de Opinión y Participación Social, A.C.

Urra, M. (2013, jan). Trabajo Social en el ámbito empresarial, proceso histórico, definiciones y tendencias. Hojas y Hablas, 10, 91-96.

Vallejo, V., y Portalanza, A. (2017). Importancia de la Gestión del Talento Humano como Estrategia para la Atracción y Retención de Docentes en las Organizaciones Educativas de Ecuador. , 24.

Viscarret, J. (2017). Modelos y métodos de Intervencion en Trabajo Social. Madrid: Alianza Editorial. 